\title{
App Review Series: Epocrates
}

\author{
Shelly Bhanot $^{1}$ (D) Arjun Sharma ${ }^{2}$
}

Published online: 18 May 2017

(C) Society for Imaging Informatics in Medicine 2017

\section{App Specs}

App Icon URL: http://www.epocrates.com/sites/default/files/ ma/static/epocrates-redesign/images/chicklet@2x.png

App Name: Epocrates

App Developer: Epocrates, Inc.

App Developer Website: http://www.epocrates.com

App Price: Free (Premium version: \$174.99/year to unlock additional features)

Apple App store URL: https://itunes.apple.com/us/app/ epocrates-reference-tools-for-healthcare-providers/ id 281935788 ? $\mathrm{mt}=8$

Google Play Store URL: https://play.google.com/store/ apps/details?id=com.epocrates\&hl=en

Category: Educational, Reference

Tags: Clinical reference, Education, Pharmacology, Healthcare

Works offline: Yes

FDA Approval: NA

Promotion Code: NA

Shelly Bhanot

sbhanot@gwu.edu

Arjun Sharma

arjunsharma33@gmail.com

1 School of Medicine and Health Sciences, George Washington University, Washington, DC, USA

2 Department of Radiology, Adventist Hinsdale Hospital, Hinsdale, IL 60521, USA

\section{Quick Review}

Overall rating (1-5): 5

Content (1-5): 5

Usability (1-5): 5

Pros Medication information and some reference material are easily accessible. Application interface is readable with a userfriendly layout. Reliable and ad-free user experience with the ability to work offline.

Cons Although a free version provides a wealth of information, premium membership allows access to additional material including illness scripts, ICD codes, reference laboratory values, and differential diagnoses.

At-A-Glance This application provides an ergonomic platform for easily accessible information regarding a large variety of medications. Facts about medications are presented simply and can be quickly comprehended.

\section{Full Review}

Intro Epocrates is a well-known web-service and mobile application used by healthcare professionals. The service initially grew in popularity as a pharmacology reference but more recently has expanded to include a variety of medical reference materials. From medical calculators to adult and pediatric reference tables, the application today spans a large variety of clinical decision support tools.

Purpose/Features/Content Expectedly, the free version of Epocrates centers on pharmacology. Most healthcare providers trained in the past decade or experienced practitioners with 
[

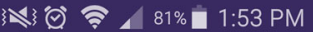

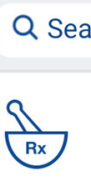

Drugs

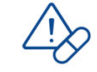

Interaction
Check

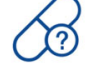

Pill ID
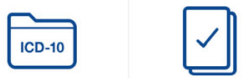

ICD-10

Guidelines

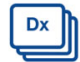

Diseases
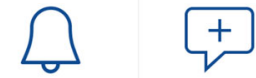

Notifications

athenaText

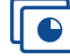

Essential
Points ${ }^{\circledR}$

-

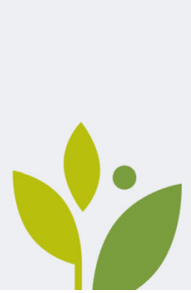

epocrates $^{\circ}+$

an athenahealth service

Fig. 1 Main menu

tech-savvy will be readily familiar with the service. Epocrates is a reliable, comprehensive, and easily navigable service for looking up information about medications: uses, dosing, contraindications, reactions, interactions, pregnancy,

\begin{tabular}{l}
\hline 0 Drugs \\
\begin{tabular}{l}
\hline carisoprodol/aspirin \\
generic \\
No Formulary Selected
\end{tabular} \\
\hline Adult Dosing \\
\hline Peds Dosing \\
\hline No Black Box Warnings \\
\hline Contraindications/Cautions \\
\hline Adverse Reactions \\
\hline Drug Interactions \\
\hline Safety/Monitoring \\
\hline Pregnancy/Lactation \\
\hline Pharmacology
\end{tabular}

Fig. 2 Sample medication menu

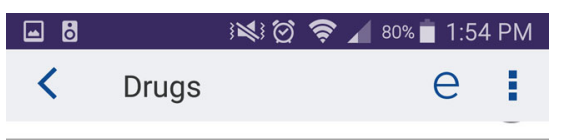

Pharmacology

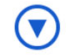

Metabolism

liver extensively; CYP450: 2B6, 2D6, 3A4 substrate;

Info: active metabolite

Excretion

urine (30\% unchanged); Half-life: $6.3-7.9 \mathrm{~h}$

(tramadol), 7.4-8.8h (active metabolite)

Subclass

Other Analgesics; Opioids

Mechanism of Action

exact mechanism of action unknown; binds to mu

opioid receptors and weakly inhibits

norepinephrine/serotonin reuptake, producing

analgesia (central opioid agonist)

Manufacturer/Pricing

Pill Pictures

Notes

Fig. 3 Sample "pharmacology" subheading

pharmacology, and more. The search function works well and is forgiving for the poor speller. With a finger tap, information from a given category can be expanded or one can easily navigate through linked keywords to other medications or diseases.

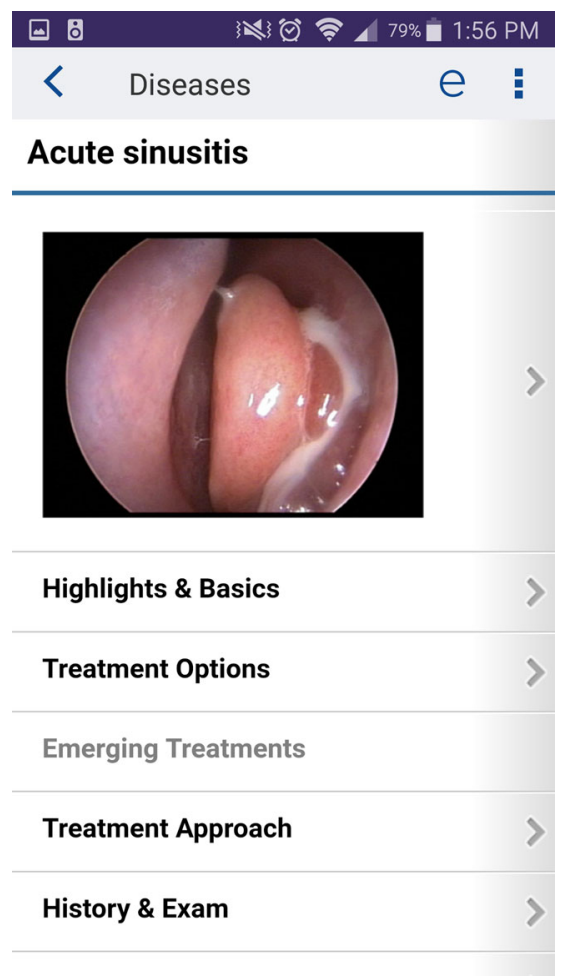

Fig. 4 Sample disease page (available as premium content) 
The professional version includes a cornucopia of decision support tools, including disease information, support for alternative medicines, infectious disease treatment, and laboratory panels and normal values. A large wealth of information is offered in a concise manner, including basic information including such as history and exam findings but also approach to diagnostic confirmation and treatment. It appears the developers took considerable effort to include as many references and calculators as possible without reaching into the obscure. The authors were frequently impressed with the amount of functionality provided. Please refer to the figures for further understanding of the application content.

Note should be made that the general radiologist is unlikely to get much use from this application unless she is directly interacting with patients on a daily basis or frequently prescribing medications.

Usability The UI is clean and professional. The user interface is excellent and works exactly as one would expect. The main menu, seen in Fig. 1, provides an attractive overview of the app's content, spanning three (free version) or four (pro) pages. As mentioned above, information is easily accessible via the application's "search" function. One level deep, information is presented in either bullet points or other shorthand.
In the authors' estimation, this format compares favorably with competitor education apps such as Up-to-Date in which information is given in paragraph form. Epocrates' quick delivery bullet points make material succinct and easily retrievable - exactly as one would want in a quick reference for the on-the-go provider (Figs. 2, 3, and 4).

Good Excellent UI and UX. Excellent port of a pharmacology reference with which many healthcare providers will already be familiar. Unexpectedly high level of functionality in clinical decision support tools. Free version provides value and a comprehensive ad-free experience.

Room for Improvement Professional version is quite expensive where institution-level access is not provided. No free trial. Small footprint.

\section{Compliance with Ethical Standards}

Sources of Support None

Conflict of Interest The authors declare that they have no conflict of interest. 